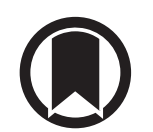

CrossMark

\title{
Size matters! Peripheral blood leukocyte telomere length and survival after critical illness
}

\author{
Florian B. Mayr (10 ${ }^{1,2}$ and Sachin Yende ${ }^{1,2}$ \\ Affiliations: 'VA Pittsburgh Healthcare System, Pittsburgh, PA, USA. ${ }^{2}$ CRISMA Center, Dept of Critical Care \\ Medicine, University of Pittsburgh School of Medicine, Pittsburgh, PA, USA. \\ Correspondence: Florian B. Mayr, VA Pittsburgh Healthcare System, University Drive C, Building 1, Rm 5A112, \\ Pittsburgh, PA 15240, USA. E-mail: florianapitt.edu
}

@ERSpublications

The association of shorter leukocyte telomere length and reduced survival in critical illness may prove crucial in our quest to improve outcomes http://bit.ly/2qDAZSq

Cite this article as: Mayr FB, Yende S. Size matters! Peripheral blood leukocyte telomere length and survival after critical illness. Eur Respir J 2020; 55: 1902114 [https://doi.org/10.1183/13993003.02114-2019].

In 2009, Elizabeth Blackburn, Jack Szostak and Carol Greider were awarded the Nobel Prize in Physiology or Medicine for their pioneering work that led to the discovery of telomeres and the enzyme complex telomerase responsible for maintaining its structure [1]. Over the past four decades, the classic view of telomeres protecting the natural ends of linear chromosomes and telomerase as telomere-terminal transferase necessary for the replication of chromosome ends has significantly evolved. Many diverse fields have matured, including the discovery of key molecular components of telomerase, implications for limits to cellular replication, and identification and characterisation of human genetic disorders that result in premature telomere shortening [2]. For example, the short telomere syndromes are a group of genetic disorders that are caused by mutations in components of the telomerase enzyme and other telomere maintenance genes [3]. The most common associated pathologies involve respiratory disorders such as idiopathic pulmonary fibrosis (IPF) and related interstitial lung disorders and severe emphysema, alone or combined with fibrosis [4-6]. Mutations in the telomerase enzyme genes are the most common cause of IPF, and the frequency of these mutations in severe, early-onset emphysema rivals the prevalence of $\alpha_{1}$-antitrypsin deficiency [4], making short telomere syndromes the most-common premature ageing disorders, with $\sim 10000$ affected individuals in the USA alone [3].

In this issue of the European Respiratory Journal, Liu et al. [7] analysed telomere length of circulating peripheral blood leukocytes (PBL-TL) in two cohorts of 1331 critically ill patients to test the hypothesis that telomere length is associated with survival in critical illness. Their primary analysis cohort consisted of 937 medical and surgical patients of whom 368 had sepsis ("VUMC cohort"). In this cohort, median PBL-TL was 6.96 kilobase $(\mathrm{kb})$ pairs (interquartile range 6.47-7.41), and shorter PBL-TLs were independently associated with worse 90-day and 1-year survival in unadjusted and adjusted analysis (adjusted for age, sex, ethnicity, smoking status and APACHE II score; 90-day survival: adjusted HR 1.3 per $1 \mathrm{~kb}$ telomere length decrease, 95\% CI 1.1-1.6; $\mathrm{p}=0.004$; for 1 -year survival: adjusted HR 1.3 per $1 \mathrm{~kb}$ telomere length decrease, 95\% CI 1.1-1.5; $\mathrm{p}=0.004$ ). Interestingly, mean PBL-TL was significantly shorter in sepsis patients compared to non-sepsis patients, and the association between 90-day and 1-year survival was even more pronounced in this subgroup. To validate these findings, secondary analyses in a "sepsis only" cohort of 394 patients were performed. Similarly, shorter PBL-TL was independently associated with 
worse 60-day survival (adjusted HR 1.6 per $1 \mathrm{~kb}$ telomere length decrease, 95\% CI 1.2-2.1; $\mathrm{p}=0.003$ ). However, there was no association between telomere length and the number of organs failing, vasopressor use, or the need for mechanical ventilation in adjusted analyses. Additional subgroup analyses suggested that shorter PBL-TL was associated with risk of progressing from mild to severe acute respiratory distress syndrome (ARDS), which again was more pronounced in sepsis patients with ARDS.

Quantitative real-time polymerase chain reaction (qPCR) of isolated DNA is commonly used to measure average PBL-TL. Despite excellent performance characteristics in this study (intra-assay coefficient of variation $(\mathrm{CV})<1 \%$ and inter-assay $\mathrm{CV}<3 \%$ ), this method has previously been criticised for its high variability rate across laboratories, in part due to its sensitivity to DNA quality and extraction methods, while also being prone to error propagation with PCR amplification [8]. Circulating leukocytes additionally have variable telomere length depending on their replicative histories, thus potentially confounding analysis of total leukocyte telomere length by fluctuations in leukocyte ratios [9]. Results may therefore not be easily reproducible across large scale multicentre studies with many different study sites and laboratories. Telomere length measurement by flow cytometry and FISH (flowFISH) has been proposed as an alternative method, which can be standardised, has reproducible and definable upper and lower normal boundaries, and has been used to inform patient care decisions for targeted clinical conditions in the hospital setting [10].

So what next? First, further research is needed to determine whether short PBL-TL is "just" a marker of poor baseline health, or if critical illness, and sepsis in particular, accelerates telomere attrition and conveys an incremental risk for adverse outcomes [11]. A recent small (and likely underpowered) study in 40 critically ill patients assessed changes in PBL-TL over 7 days and reported shortening in PBL-TL in 21 out of 40 patients, lengthening in 11 out of 40 and no change in the remaining eight patients, but failed to demonstrate any association with clinical outcomes [12]. In addition, the current study by Liu et al. [7] did not find any statistically significant association of PBL-TL with severity of illness, i.e. the number of organs failing, need for vasopressors and mechanical ventilation, respectively.

Alternatively, PBL-TL in critically ill patients may be a marker of pre-existing chronic health and immune competence. For example, in a large prospective Danish cohort of 75309 people who were followed for up to 23 years, individuals within the shortest quartile of PBL-TL had a higher risk of infection and pneumonia-related hospitalisations than individuals with longer PBL-TL. Understanding the relationship between PBL-TL and immune phenotype is particularly important in patients with sepsis who frequently have sustained immunosuppression that predisposes them to recurrent infections and infection-related hospitalisations [13]. The lack of cytokine data, which would be needed to better understand the relationship between telomere length and immune phenotype, is a limitation of the current study and should be on the future research agenda. If short PBL-TL is associated with an immunocompromised phenotype, future studies to explore PBL-TL as a predictive biomarker for immunomodulatory therapies may be warranted. Second, the severity of many chronic health conditions is associated with telomere length including obesity, diabetes, heart failure, and chronic obstructive lung disease [14-17]. These diseases are highly prevalent among patients with sepsis, and future studies should provide more clinical detail about pre-existing health conditions to minimise the risk of residual confounding.

In conclusion, the association of shorter leukocyte telomere length and reduced survival in critical illness, and sepsis in particular, is an intriguing finding at the intersection of immune competence, chronic health and cellular senescence that may prove crucial in our quest to improve outcomes after critical illness.

Conflict of interest: F.B. Mayr reports grants from National Institutes of Health and Department of Veterans Affairs, outside the submitted work. S. Yende reports personal fees from Atox Bio, grants from Bristol-Myers Squibb and Roche, outside the submitted work.

Support statement: F.B. Mayr is currently supported by a VISN4 Competitive Career Development Fund from the US Department of Veterans Affairs and NIH K23GM132688. Funding information for this article has been deposited with the Crossref Funder Registry.

\section{References}

1 Varela E, Blasco M. 2009 Nobel Prize in Physiology or Medicine: telomeres and telomerase. Oncogene 2010; 29: 1561-1565.

Shay JW, Wright WE. Telomeres and telomerase: three decades of progress. Nat Rev Genet 2019; 20: 299-309. Armanios M, Blackburn EH. The telomere syndromes. Nat Rev Genet 2012; 13: 693-704.

Stanley SE, Chen J, Podlevsky JD, et al. Telomerase mutations in smokers with severe emphysema. J Clin Invest 2015; 125: 563-570.

5 Alder JK, Chen J, Lancaster L, et al. Short telomeres are a risk factor for idiopathic pulmonary fibrosis. Proc Natl Acad Sci USA 2008; 105: 13051-13056. 
6 Armanios MY, Chen J, Cogan JD, et al. Telomerase mutations in families with idiopathic pulmonary fibrosis. N Engl J Med 2007; 356: 1317-1326.

7 Liu S, Wang C, Green G, et al. Peripheral blood leukocyte telomere length is associated with survival of sepsis patients. Eur Respir J 2020; 55: 1901044.

8 Dagnall CL, Hicks B, Teshome K, et al. Effect of pre-analytic variables on the reproducibility of qPCR relative telomere length measurement. PLoS One 2017; 12: e0184098.

9 Aubert G, Baerlocher GM, Vulto I, et al. Collapse of telomere homeostasis in hematopoietic cells caused by heterozygous mutations in telomerase genes. PLoS Genet 2012; 8: e1002696.

10 Alder JK, Hanumanthu VS, Strong MA, et al. Diagnostic utility of telomere length testing in a hospital-based setting. Proc Natl Acad Sci USA 2018; 115: E2358-E2365.

11 Ilmonen P, Kotrschal A, Penn DJ. Telomere attrition due to infection. PLoS One 2008; 3: e2143.

12 Zribi B, Uziel O, Lahav M, et al. Telomere length changes during critical illness: a prospective, observational study. Genes (Basel) 2019; 10: E761.

13 Hotchkiss RS, Monneret G, Payen D. Immunosuppression in sepsis: a novel understanding of the disorder and a new therapeutic approach. Lancet Infect Dis 2013; 13: 260-268.

14 Testa R, Olivieri F, Sirolla C, et al. Leukocyte telomere length is associated with complications of type 2 diabetes mellitus. Diabet Med 2011; 28: 1388-1394.

15 van der Harst P, van der Steege G, de Boer RA, et al. Telomere length of circulating leukocytes is decreased in patients with chronic heart failure. J Am Coll Cardiol 2007; 49: 1459-1464.

16 Moreno-Navarrete JM, Ortega F, Sabater M, et al. Telomere length of subcutaneous adipose tissue cells is shorter in obese and formerly obese subjects. Int J Obes 2010; 34: 1345-1348.

17 Jin M, Lee E, Ra S, et al. Relationship of absolute telomere length with quality of life, exacerbations, and mortality in COPD. Chest 2018; 154: 266-273. 